\title{
AVALIAÇÃO DA UTILIZAÇÃO DO COQUE BREEZE NO PROCESSO DE PELOTIZAÇÃO DE MINÉRIO DE FERRO*
}

\author{
Camila Luzia Lira Duarte ${ }^{1}$ \\ Frederico Mayerhofer ${ }^{2}$ \\ Gleison Vinicius Ricardo ${ }^{3}$ \\ Luis Torres Dantas ${ }^{4}$ \\ Marcos Barros ${ }^{5}$ \\ Silvio Maranha ${ }^{6}$ \\ Tiago Gonçalves da Silva ${ }^{7}$
}

\section{Resumo}

O processo de pelotização utiliza o combustível sólido com intuito de gerar energia térmica que será transferida para a pelota de forma a alcançar uma queima uniforme. Na usina de Vargem Grande normalmente o antracito é utilizado como combustível sólido. Com objetivo de testar diferentes fontes de combustível sólido, um teste industrial de com coque breeze substituindo o antracito foi realizado. Como o coque breeze possui menor teor de matéria volátil, cinzas ei maior teor de carbono fixo, o consumo de gás natural no forno pôde ser reduzido. Além disto, o coque breeze apresentou um perfil de combustão mais favorável à qualidade da pelota resultando em ganho nos resultados de compressão. Dessa forma, a viabilidade econômica e operacional da substituição do antracito pelo coque breeze foi positiva. Palavras-chave: Coque breeze; Combustível sólido; Pelotização.

\section{INDUSTRIAL TRIAL WITH COKE BREEZE IN THE PELLETIZING PROCESS Abstract}

The pelletizing process for hematite ores must uses solid fuel inside the green pellets in addition to the natural gas to generate the thermal energy which is transferred to the firing pellet, aiming a homogeneous indurating process. In order to test a different source of solid fuel, industrial trial with coke breeze was carried out replacing the anthracite that is often used. Since coke breeze contains smaller amount of volatile material, ashes and higher fixed carbon, the gas consumption in the furnace could be reduced. Moreover, the pellets with coke breeze have shown better combustion pattern which results in higher compression strength. Therefore, both the economical and operational figures have shown positive outcomes when coke breeze is used instead of anthracite.

Keywords: Coke breeze; Solid fuel; Pelletizing.

1 Engenheira Química, Bacharel, Engenheira de Processo, Departamento de Pelotização, Vale, Nova Lima, Minas Gerais - Brasil.

2 Engenheiro Metalurgista, Bacharel, Gerente de processo, Departamento de Pelotização, Vale, Vitória, Espirito Santo - Brasil

3 Técnico em Mineração, Técnico, Técnico em Controle de Processo, Departamento de Pelotização, Vale, Nova Lima, Minas Gerais - Brasil.

4 Engenheiro Metalurgista, Bacharel, Engenheiro de Processo, Departamento de Pelotização, Vale, Nova Lima, Minas Gerais - Brasil.

5 Engenheiro de Minas, Bacharel, Engenheiro de Processo, Departamento de Pelotização, Vale, Nova Lima, Minas Gerais - Brasil.

6 Engenheiro Metalurgista, Bacharel, Engenheiro Master, Marketing e Vendas de Minério de Ferro, Vale, Nova Lima, Minas Gerais - Brasil.

7 Técnico em Metalurgia, Técnico, Técnico em Controle de Processo, Departamento de Pelotização, Vale, Nova Lima, Minas Gerais - Brasil. 


\section{INTRODUÇÃO}

O carvão coque é um subproduto do carvão mineral, é obtido através do processo denominado de coqueificação, onde o carvão mineral é submetido a altas temperaturas na ausência de oxigênio. O coque aparece ao final da queima, na forma de um resíduo sólido e poroso.

O coque é utilizado como matéria prima combustível nos altos-fornos, na produção de aço.

No processo de Pelotização podem ser utilizados vários tipos de carvões como aditivos energéticos sólidos dependendo de estudos de influências na qualidade e facilidades para o processo, além da disponibilidade para o fornecimento.

Dentre eles podemos citar:

- Carvão Vegetal

- Carvão Mineral-Normalmente antracítico

- Finos de coque

- Coque verde de petróleo

Os combustíveis sólidos propiciam uma boa homogeneização da distribuição de energia no leito de pelotas dentro do forno de grelha móvel principalmente

no final da queima, onde a queima do carvão existente nas camadas inferiores de pelotas cruas tende a equalizar a queima e a qualidade das pelotas.

Possibilitam aumentos significativos de produtividade porque desde que se tenha um bom controle das temperaturas que as pelotas estão atingindo, é possível impor um maior ritmo á produção pelo aumento da velocidade da grelha móvel.

Os combustíveis sólidos podem introduzir elementos de ganga na pelota. Dependendo do tipo de carvão utilizado algumas impurezas podem ser introduzidas na pelota e estas podem ou não influenciar a qualidade. Dessa forma o carvão que melhor se ajusta a essa condição deve ser escolhido. Por exemplo, os carvões antraciticos podem ter níveis de sílica inadequados e os carvões vegetais normalmente tem nível de álcalis muito altos.

O controle de adição deve ser rigoroso para não causar perda de qualidade da pelota. Qualquer descontrole na adição de carvão pode influenciar negativamente a qualidade da pelota. Normalmente essas interferências são observáveis na compressão e no tamboramento da pelota.

\section{OBJETIVOS}

Testar o coque breeze como fonte alternativa de combustível sólido no processo de pelotização.

\section{DESENVOLVIMENTO}

\subsection{Dados do teste}

A realização do teste industrial de utilização do coke breeze teve o objetivo de realizar avaliação econômica e operacional do uso deste combustível sólido em subsituição ao antracito com foco em qualidade, produtividade e custo.

O teste industrial de utilização operacional do coque breeze foi realizado em 2 etapas, compreendendo 2 períodos entre os dias 14/11 e 19/11/14 e 28/01 a $31 / 01 / 15$. Esta interrupção foi necessária pois a elevada umidade do coque breeze recebido levou à queda da taxa de produção e do rendimento operacional da 
moagem de insumos. Consequentemente, restringindo a taxa de produção da usina pelo baixo estoque de mix.

Foram consumidos no teste 3.183 toneladas de coque breeze.

\subsection{Preparação}

Foi feita caracterização do coke breeze e do antracito no Centro tecnológico de ferrosos da Vale e no laboratório químico da usina.

$\mathrm{Na}$ caracterização individual de 2 amostras de antracito e 2 amostras de coque breeze (amostras coletadas em períodos distintos) foram realizadas as seguintes analises:

- Distribuição granulométrica

- HGI

- Porosidade

- Termogravimetria

- Análise Química

$\mathrm{Na}$ caracterização das pelotas queimadas com coque breeze foram realizadas as seguintes análises:

- Análise macroscópica

- Porosimetria por intrusão de mercúrio

- Análise de imagens de microscopia óptica

\subsection{Desenvolvimento}

$\mathrm{Na}$ usina de pelotização de Vargem Grande utiliza-se uma mistura de calcário e combustível sólido chamada de mix. O mix é formado na alimentação do moinho de insumos da pelotização, onde insumo bruto é moído para atingir granulometria adequada ao processo de pelotização.

A moagem foi realizada em ambos os moinhos de insumos sendo moinho vertical de rolos e moinho de bolas.

A usina de pelotização de Vargem Grande tem como parâmetro crítico de qualidade os resultados de compressão da pelota. Foi feita comparação entre os resultados de qualidade da pelota queimada com uso de antracito e coque breeze. Além disto, era esperado que fosse possível elevar a dosagem de combustível sólido e consequentemente carbono fixo com intuito de economizar gás natural no forno.

Foi planejado elevar a dosagem de carbono fixo para verificar o impacto nos resultados de compressão e no consumo de gás natural.

No teste foi utilizada uma proporção no mix de 50/50 de coque breeze e calcário No processo de queima foram mensurados os resultados de produtividade de grelha, consumo de gás e qualidade.

Foi realizada análise de viabilidade financeira da substiuição do antracito pelo coque breeze.

\section{RESULTADOS E DISCUSSÃO}

\section{Caracterização}

Na tabela abaixo são apresentados os resultados da analise imediata do antracito e do coque breeze. 
$46^{\circ}$ Redução

$17^{\circ}$ Minério de Ferro

$4^{\circ}$ Aglomeração

ISSN 2176-3135

\begin{tabular}{|l|c|c|c|c|c|c|c|}
\hline \multicolumn{1}{|c|}{ Tipo } & $\%$ Cfixo & $\%$ C total & $\%$ cinzas & PCI (cal/g) & Umidade & MV(\%) & S (\%) \\
\hline Coque 1 etapa & 78,40 & 82,06 & 17,92 & 6192 & 11,59 & 3,72 & 0,60 \\
\hline $\begin{array}{l}\text { Coque 2etapa } \\
\text { 21-01-15 }\end{array}$ & 75,5 & 78,43 & 16,01 & 6803 & - & 8,49 & 1,36 \\
\hline $\begin{array}{l}\text { Coque 2 etapa } \\
\text { 28-01-15 }\end{array}$ & 78,8 & 81,58 & 17,69 & 6138 & 8,14 & 3,55 & 0,73 \\
\hline Antracito típico & 71,77 & 74,16 & 18,84 & 6426 & 6,51 & 9,36 & 0,80 \\
\hline
\end{tabular}

Tabela 1. Resultados de análise imediata do antracito e amostras de coque breeze.

Em siderúrgicas, a fim de garantir o bom desempenho do $\mathrm{AF}$, o coque produzido nas coquerias geralmente apresenta teor de matéria volátil inferior a $2 \%$, teores de cinza de no máximo $11,5 \%$ e por consequência teores de carbono fixo acima de $85 \%$.

\begin{tabular}{|c|c|c|c|c|c|c|}
\hline Amostrac & \multicolumn{3}{|c|}{ Antracito (amostra1) } & \multicolumn{3}{c|}{ Coque Breeze (amostra 1) } \\
\hline $\begin{array}{c}\text { Abertura } \\
(\mathrm{mm})\end{array}$ & $\begin{array}{c}\text { Retida } \\
\text { Simples (\%) }\end{array}$ & $\begin{array}{c}\text { Retida } \\
\text { Acumulada } \\
(\%)\end{array}$ & $\begin{array}{c}\text { Passante } \\
(\%)\end{array}$ & $\begin{array}{c}\text { Retida } \\
\text { Simples }(\%)\end{array}$ & $\begin{array}{c}\text { Retida } \\
\text { Acumulada } \\
(\%)\end{array}$ & $\begin{array}{c}\text { Passante } \\
(\%)\end{array}$ \\
\hline 76,00 & 0,00 & 0,00 & 100,00 & 0,00 & 0,00 & 100,00 \\
\hline 50,00 & 0,00 & 0,00 & 100,00 & 0,00 & 0,00 & 100,00 \\
\hline 25,00 & 0,00 & 0,00 & 100,00 & 0,00 & 0,00 & 100,00 \\
\hline 12,50 & 0,59 & 0,59 & 99,41 & 2,86 & 2,86 & 97,14 \\
\hline 6,30 & 16,58 & 17,16 & 82,84 & 10,88 & 13,73 & 86,27 \\
\hline 4,00 & 17,29 & 34,45 & 65,55 & 13,24 & 26,97 & 73,03 \\
\hline 2,83 & 7,54 & 42,00 & 58,00 & 7,69 & 34,66 & 65,34 \\
\hline 1,41 & 24,02 & 66,01 & 33,99 & 15,23 & 49,89 & 50,11 \\
\hline 0,50 & 17,01 & 83,02 & 16,98 & 19,28 & 69,17 & 30,83 \\
\hline 0,25 & 6,70 & 89,72 & 10,28 & 13,37 & 82,54 & 17,46 \\
\hline 0,15 & 3,40 & 93,11 & 6,89 & 8,58 & 91,12 & 8,88 \\
\hline$-0,15$ & 6,89 & 100,00 & 0,00 & 8,88 & 100,00 & 0,00 \\
\hline
\end{tabular}

Tabela 2. Distribuição granulométrica do antracito e coque breeze.

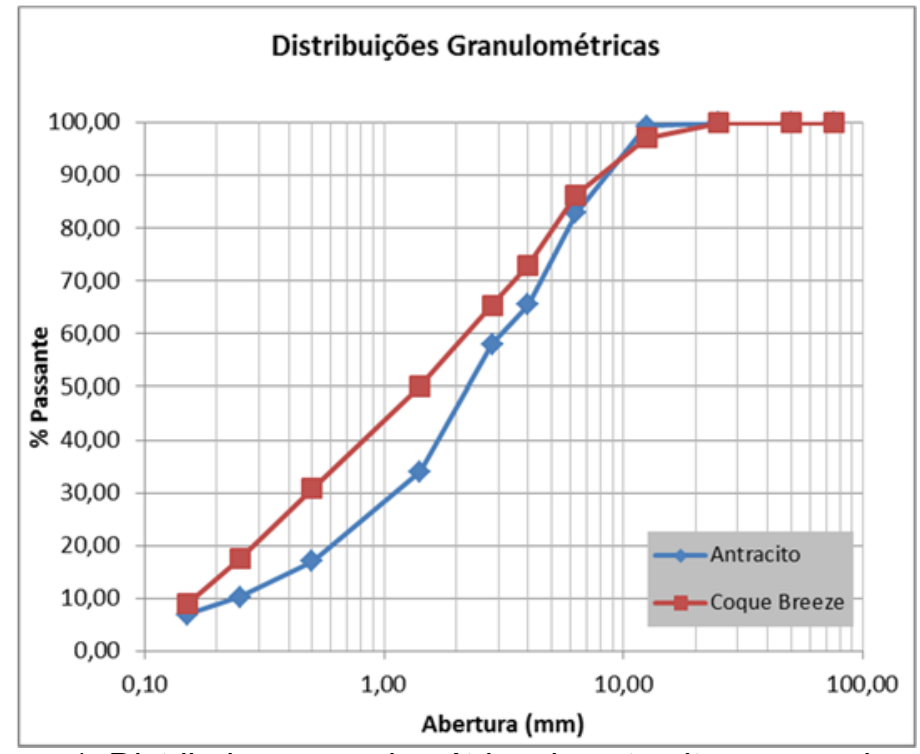

Figura 1. Distribuição granulométrica do antracito e coque breeze 
Como era de se esperar, o antracito apresentou granulometria mais grossa que o coque breeze, pois o coque já é adquirido do fornecedor numa granulometria mais fina.

Foi observado que $13,73 \%$ das partículas do coque breeze ficaram retidas na peneira $+6,3 \mathrm{~mm}$, o que pode significar contaminação do material com outro tipo de combustível.

Algumas das pedras de coque breeze maiores que $6,3 \mathrm{~mm}$ foram coletadas e avaliadas visualmente (figura 2). Foi possível identificar pedras de antracito e/ou carvão betuminoso misturado ao coque, corroborando para a suspeita de contaminação.

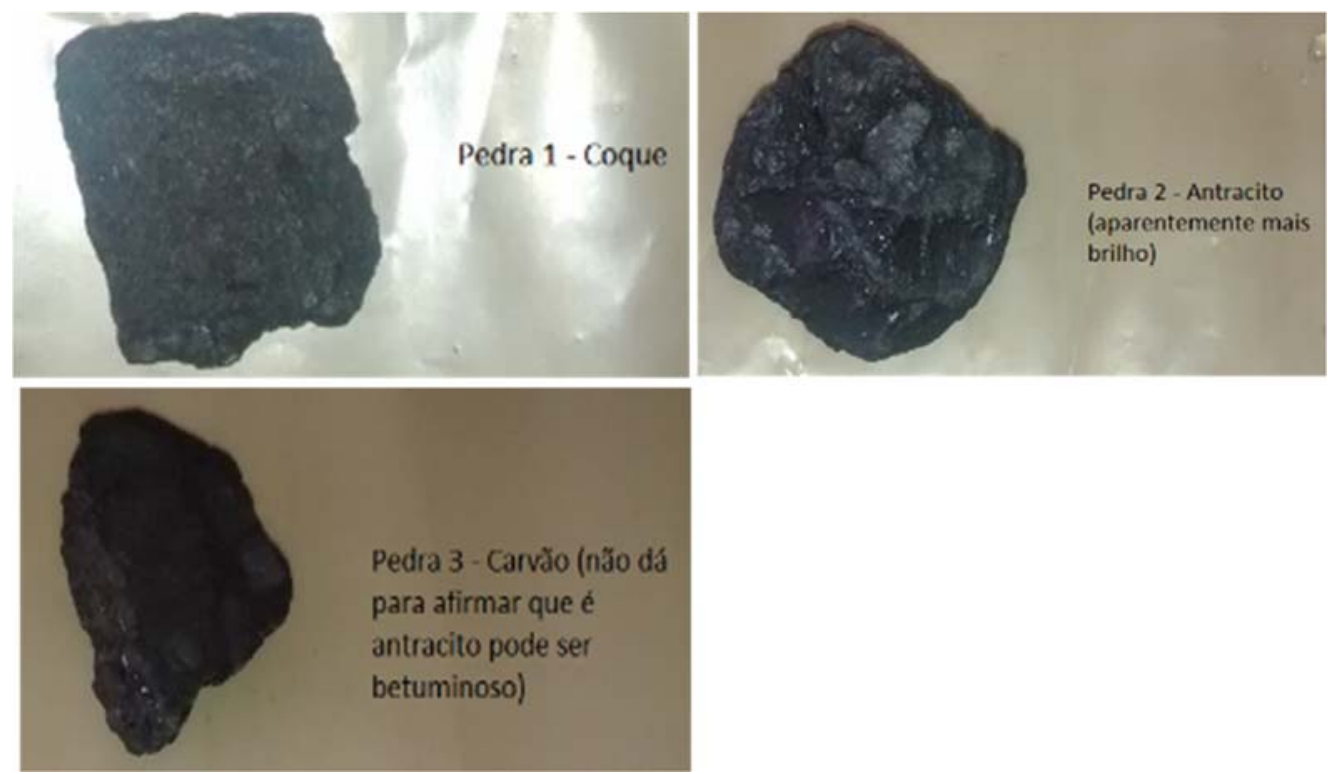

Figura 2. Análise visual do coque breeze bruto

Pode ser observado que o coque breeze apresentou maior porosidade e maiores teores de umidade (inerente, superficial e total) que o antracito.

\begin{tabular}{|c|c|c|}
\hline Amostra primeiro lote & Antracito (AK) & Coque Breeze (CB) \\
\hline Porosidade (\%) & 19,5 & 34,0 \\
\hline Umidade Superficial (\%) & 7,69 & 11,27 \\
\hline Umidade Inerente (\%) & 0,99 & 1,69 \\
\hline Umidade Total (\%) & 8,68 & 12,96 \\
\hline \hline
\end{tabular}

Tabela 3. Resultados de análise do antracito e coque breeze

A umidade inerente fica localizada nos poros do carvão. Quanto mais poros, maior será a umidade inerente do combustível. A umidade superficial é adquirida quando o combustível é processado nas plantas de beneficiamento, transportado e estocado em pátios. A soma destas duas umidades é a umidade total. 


\begin{tabular}{|c|c|c|}
\hline Amostra & $\begin{array}{c}\text { Antracito } \\
\text { (AK) }\end{array}$ & $\begin{array}{c}\text { Coque } \\
\text { Breeze (CB) }\end{array}$ \\
\hline Umidade Total (\%) & 8,17 & 12,57 \\
\hline HGl analisado 1 & 58,47 & 40,97 \\
\hline HGl analisado 2 & 48,27 & 50,32 \\
\hline
\end{tabular}

Tabela 4. Resultados de análise do antracito e coque breeze

O HGI é um índice que mede a facilidade com a qual um combustível pode ser moído. Esse índice é expresso em números que variam entre 30 e 110 e quanto mais elevado o $\mathrm{HGI}$ maior a facilidade de pulverização do combustível.

O HGI do coque breeze do primeiro lote foi inferior ao do antracito, o que era de se esperar, pois o coque é um material mais duro. Enquanto o Gl do segundo lote amostras foi muito semelhante ao do antracito. Isso pode ser um indicio que esse lote estava mais contaminado com antracito que o primeiro.

A propriedade abrasão também deve ser levada em consideração, uma vez que materiais muito abrasivos levam a maiores desgastes das superfícies de britagem/moagem e até de equipamentos de transporte. De acordo com a literatura a abrasão do antracito é $686 \mathrm{mg}$ e do coque é $2.506 \mathrm{mg}$.

Pela técnica de termobalança é possível avaliar o perfil de combustão dos combustíveis. Várias temperaturas podem ser extraídas dos perfis de combustão das amostras, as quais são:

Tc: temperatura na qual a perda de massa por aquecimento compensa o ganho de massa devido à quimissorção a temperaturas baixas;

Ti: temperatura de início da combustão, temperatura na qual a perda de massa é $20 \%$ da perda máxima1. Carvões de determinados ranks apresentam antes do início da combustão um processo de quimisorção de oxigênio em sua estrutura química (que corresponde no gráfico a um aumento de massa), o que impede a determinação exata da temperatura da primeira perda de massa;

Tp: temperatura em que há o máximo de reatividade;

Tf: temperatura final de combustão.

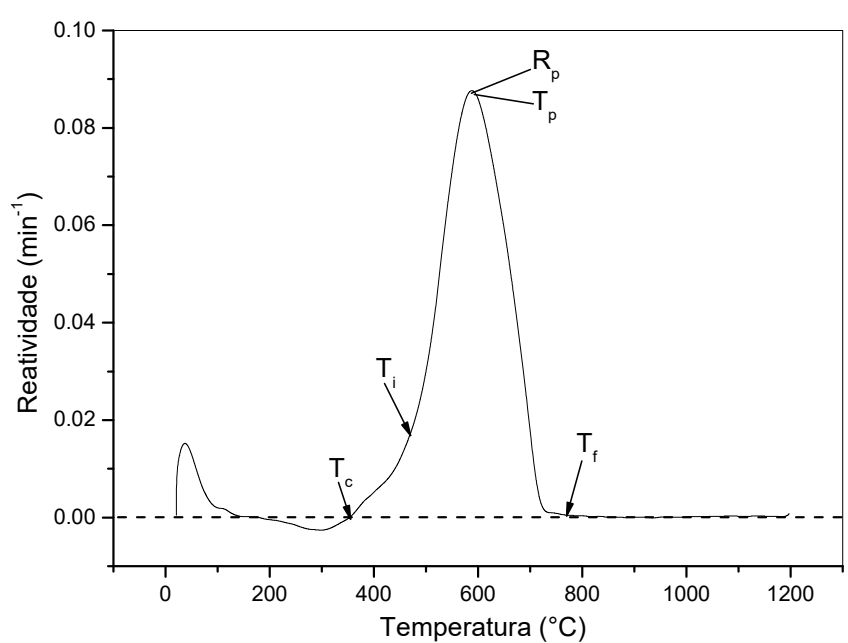

\begin{tabular}{|c|c|c|c|c|c|}
\hline $\begin{array}{c}\text { Amostra primeiro } \\
\text { lote }\end{array}$ & $\mathbf{T}_{\mathbf{C}}\left({ }^{\circ} \mathbf{C}\right)$ & $\mathbf{T}_{\mathbf{i}}\left({ }^{\circ} \mathbf{C}\right)$ & $\mathbf{T}_{\mathbf{f}}\left({ }^{\circ} \mathbf{C}\right)$ & $\mathbf{T}_{\mathbf{p}}\left({ }^{\circ} \mathbf{C}\right)$ & $\begin{array}{c}\mathbf{R}_{\mathrm{p}} \\
\left(\mathbf{m i n}^{-1}\right)\end{array}$ \\
\hline Antracito & 361 & 474 & 690 & 576 & 0,1079 \\
\hline Coque Breeze & 386 & 528 & 861 & 653 & 0,0780 \\
\hline
\end{tabular}

Figura 3. Gráfico e tabela com resultados da termobalança do antracito e coque breeze 
De acordo com os estudos que foram desenvolvidos no CTF, os principais parâmetros do combustível sólido que afetam a qualidade da pelota (resistência à compressão) são (i) a temperatura inicial de queima (Ti), quanto menor pior a RC, e (ii) a reatividade do combustível $(\mathrm{Rp})$, quanto menor maior a $\mathrm{RC}$.

Para estimar o nível de contaminação do coque breeze foi feita uma ponderação realizada através do parâmetro matéria volátil indica um nível de contaminação em torno de $20 \%$ de Antracito (ou não CB) neste lote.

\section{$\underline{\text { Resultados teste }}$}

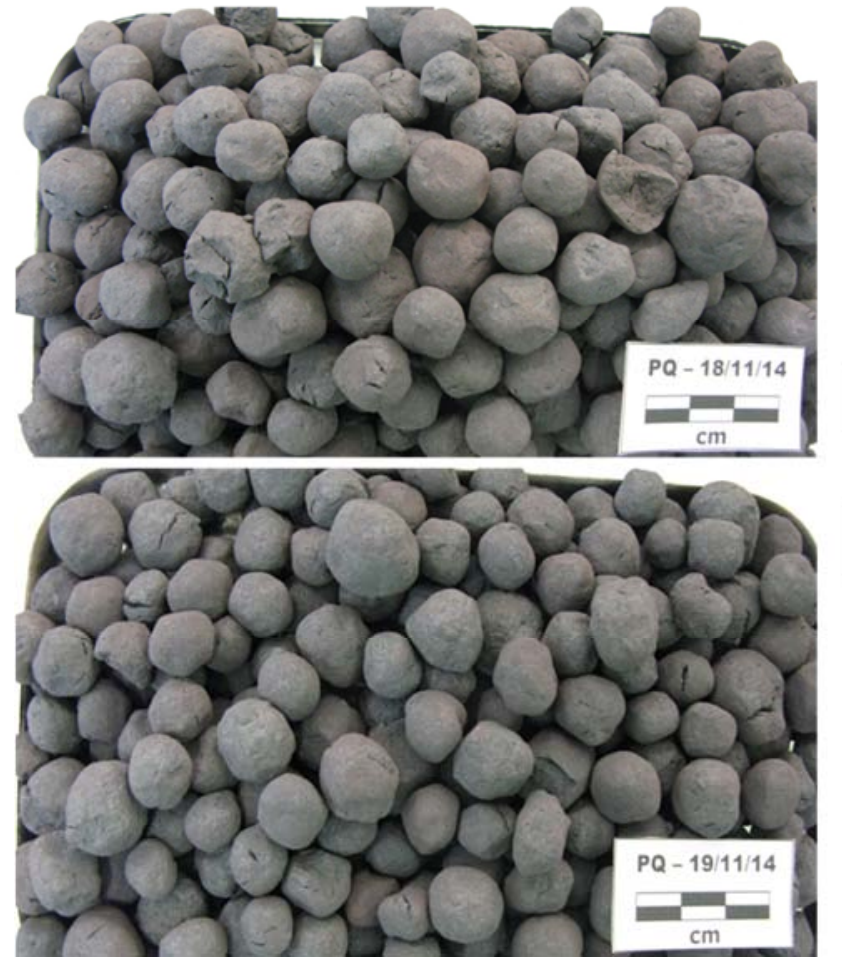

\begin{tabular}{|c|c|c|c|}
\hline Característica & PQ18/11/14 & PQ19/11/14 & AF40 \\
\hline Normal, \% & 29 & 30 & 31 \\
\hline Trinca fina, \% & 23 & 26 & 12 \\
\hline Trinca grossa, \% & 20 & 14 & 23 \\
\hline Geminada, \% & 2 & 3 & 0 \\
\hline Satélite, \% & 6 & 5 & 6 \\
\hline Fragmento, \% & 20 & 22 & 28 \\
\hline Total & 100 & 100 & 100 \\
\hline
\end{tabular}

Figura 4. Foto das amostras de pelota do teste e tabela com análise de trincas das amostras do teste e referência

A análise macroscópica das pelotas com coque breeze mostrou que ambas possuem características similares. Por outro lado, a proporção de pelotas com trincas finas diminuiu enquanto que a quantidade de pelotas com trinca grossa aumentou em comparação com uma pelota referência.

A proporção de fragmentos na pelota com coque breeze também é menor se comparada com a pelota referência.

\begin{tabular}{|c|c|c|}
\hline Pelota & Porosidade $\mathbf{H g}(\mathbf{\%})$ & Diâmetro médio dos poros $(\boldsymbol{\mu m})$ \\
\hline $\mathrm{PQ} \mathrm{18/11/14}$ & 31,7 & 8,7 \\
\hline PQ 19/11/14 & 34,5 & 8,9 \\
\hline Referência & 35,7 & 9,2 \\
\hline
\end{tabular}

Tabela 5. Resultados de análise do antracito e coque breeze

A porosidade da pelota PQ 18/11/14 é ligeiramente menos porosa $(31,7 \%)$ se comparada a PQ 19/11/14 (34,5\%). Contudo, o diâmetro médio dos poros é similar 
para ambas as pelotas. A porosidade e o diâmetro dos poros das pelotas feitas com coque breeze são menores que da pelota referência feita com antracito.

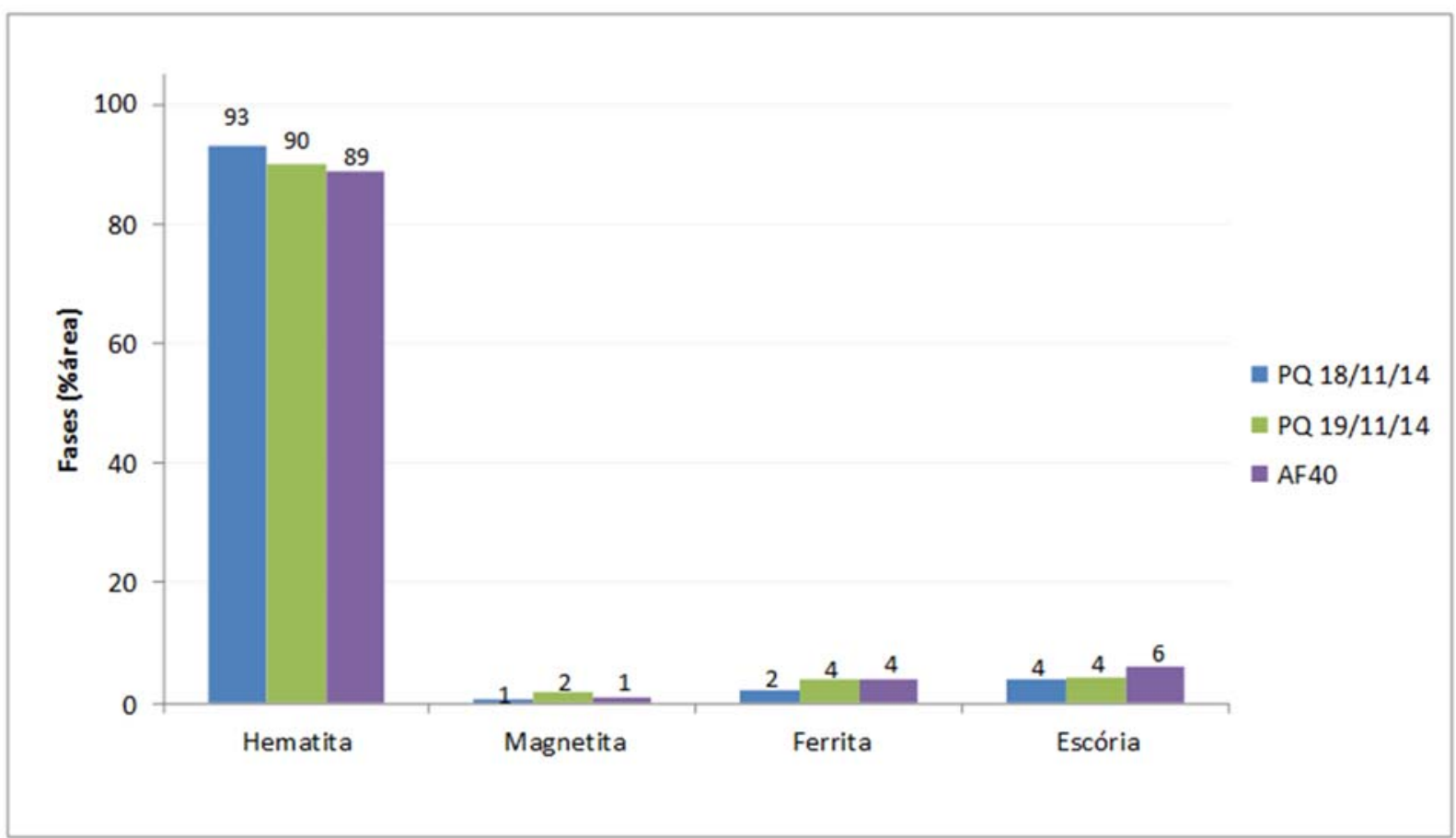

Figura 5. Análise de composição das amostras do teste e referência

De maneira geral, a composição das pelotas é similar, com pequenas variações no teor de hematita em favor dos teores de ferrita e escória.

\begin{tabular}{|c|c|c|c|c|c|c|}
\hline Data & $\begin{array}{c}\text { Taxa } \\
\text { alimentação } \\
\text { grelha (t/h) }\end{array}$ & $\begin{array}{c}\text { Compressão } \\
\text { (daN) }\end{array}$ & $\begin{array}{c}\text { Carbono } \\
\text { fixo } \\
(\%)\end{array}$ & $\begin{array}{c}\text { Consumo } \\
\text { de gás } \\
(\mathbf{N m} 3 / \mathbf{t})\end{array}$ & $\begin{array}{c}\text { Consumo } \\
\text { coque } \\
\text { (kg/t) }\end{array}$ & $\begin{array}{c}\text { Energia } \\
\text { Térmica } \\
\text { (Mcal/t) }\end{array}$ \\
\hline $14 / 11 / 14$ & 985 & 310 & 1,05 & 11,91 & 13,8 & 191 \\
\hline $15 / 11 / 14$ & 981 & 309 & 1,17 & 12,07 & 15,3 & 201 \\
\hline $16 / 11 / 14$ & 905 & 289 & 1,18 & 12,00 & 15,4 & 200 \\
\hline $17 / 11 / 14$ & 1016 & 280 & 1,25 & 11,17 & 16,4 & 201 \\
\hline $18 / 11 / 14$ & 847 & 293 & 1,25 & 11,38 & 16,3 & 200 \\
\hline $19 / 11 / 14$ & 681 & 329 & 1,20 & 12,70 & 15,3 & 204 \\
\hline $28 / 01 / 15$ & 862 & 303 & 1,08 & 12,29 & 14,7 & 212 \\
\hline $29 / 01 / 15$ & 785 & 332 & 1,07 & 11,91 & 14,7 & 206 \\
\hline $30 / 01 / 15$ & 884 & 286 & 1,21 & 11,21 & 16,4 & 217 \\
\hline $31 / 01 / 15$ & 902 & 293 & 1,22 & 11,19 & 16,6 & 218 \\
\hline
\end{tabular}

Tabela 6. Resultados operacionais do teste

Algumas restrições ao aumento de produção ocorreram durante o teste:

- Na $1^{\text {a }}$ etapa (a partir do dia 18/11) houve restrição na produção causada pelo baixo estoque de mix

- Na $2^{\mathrm{a}}$ etapa a produção ficou restrita pelo baixo fornecimento de feed 
Já nas primeiras horas do teste uma elevação acentuada da compressão foi observada, conforme tabela abaixo.

\begin{tabular}{|c|c|c|c|c|}
\hline Data & $\begin{array}{c}\text { Taxa } \\
\text { alimentação } \\
\text { grelha (t/h) }\end{array}$ & $\begin{array}{c}\text { Carbono } \\
\text { fixo }(\%)\end{array}$ & $\begin{array}{c}\text { Consumo } \\
\text { gás } \\
\text { (Nm3/t) }\end{array}$ & $\begin{array}{c}\text { Compressão } \\
\text { (daN) }\end{array}$ \\
\hline $13 / 11 / 201423: 00$ & 947 & 1,09 & 11,33 & 308 \\
\hline $14 / 11 / 201401: 00$ & 933 & 1,10 & 11,57 & 259 \\
\hline $14 / 11 / 201403: 00$ & 952 & 1,06 & 11,86 & 258 \\
\hline $14 / 11 / 201405: 00$ & 938 & 1,02 & 12,53 & 333 \\
\hline $14 / 11 / 201407: 00$ & 962 & 1,02 & 12,84 & 333 \\
\hline $14 / 11 / 201409: 00$ & 982 & 0,95 & 12,94 & 359 \\
\hline $14 / 11 / 201411: 00$ & 976 & 1,01 & 12,21 & 340 \\
\hline
\end{tabular}

Tabela 7. Resultados operacionais nas primeiras horas do teste

Para os períodos analisados o uso do coque breeze, levou em média, a maiores resultados de compressão, abrasão e finos, o que era esperado pelo perfil de combustão do coque breeze mais favorável à queima das pelotas.
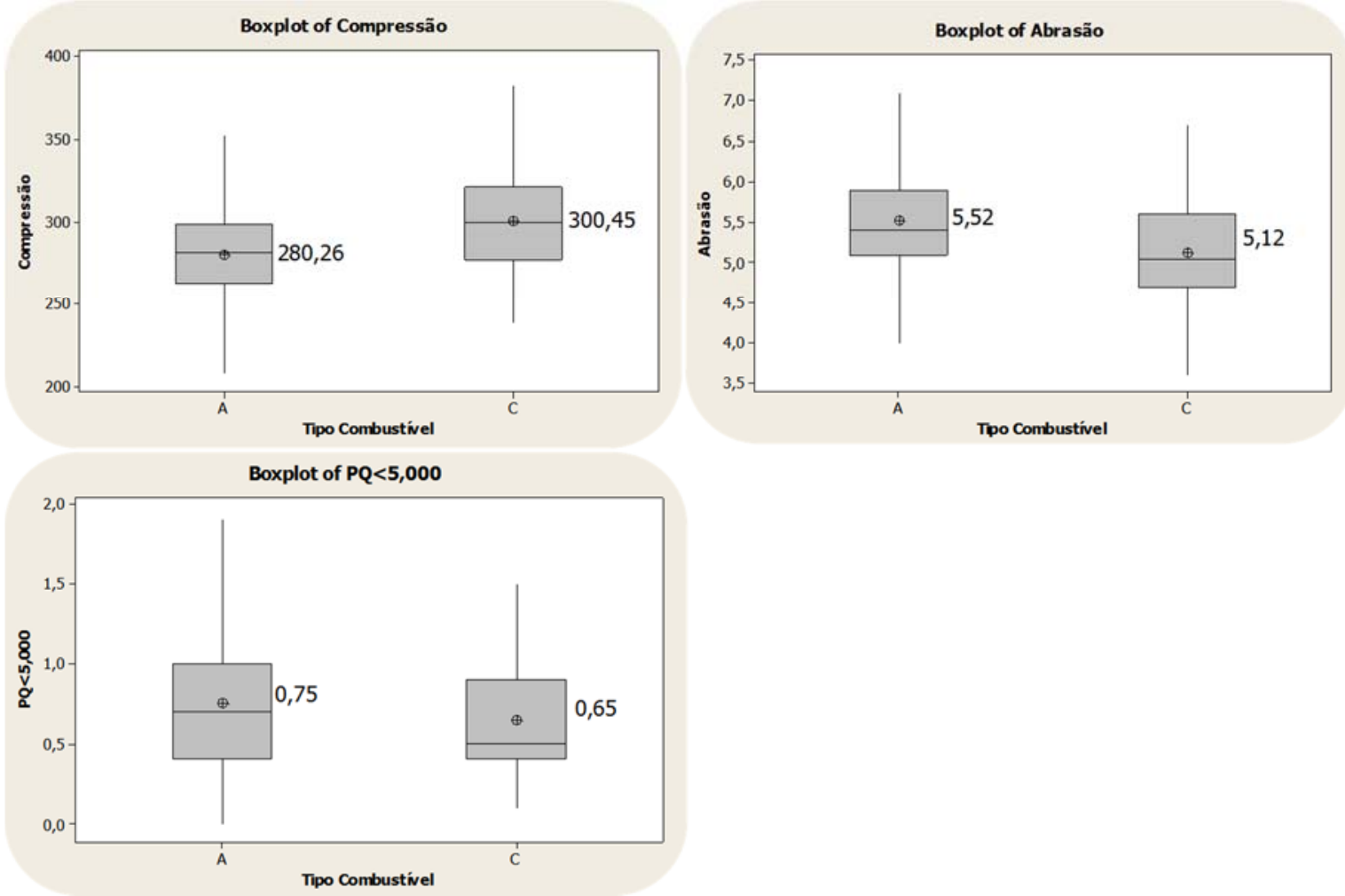

Figura 6. Boxplot para resultados de qualidade do uso de antracito (A) e coque breeze (C). Período compreendido entre 01/10/2014 e 01/02/2015

Em média, houve um aumento da compressão de 20 daN. 

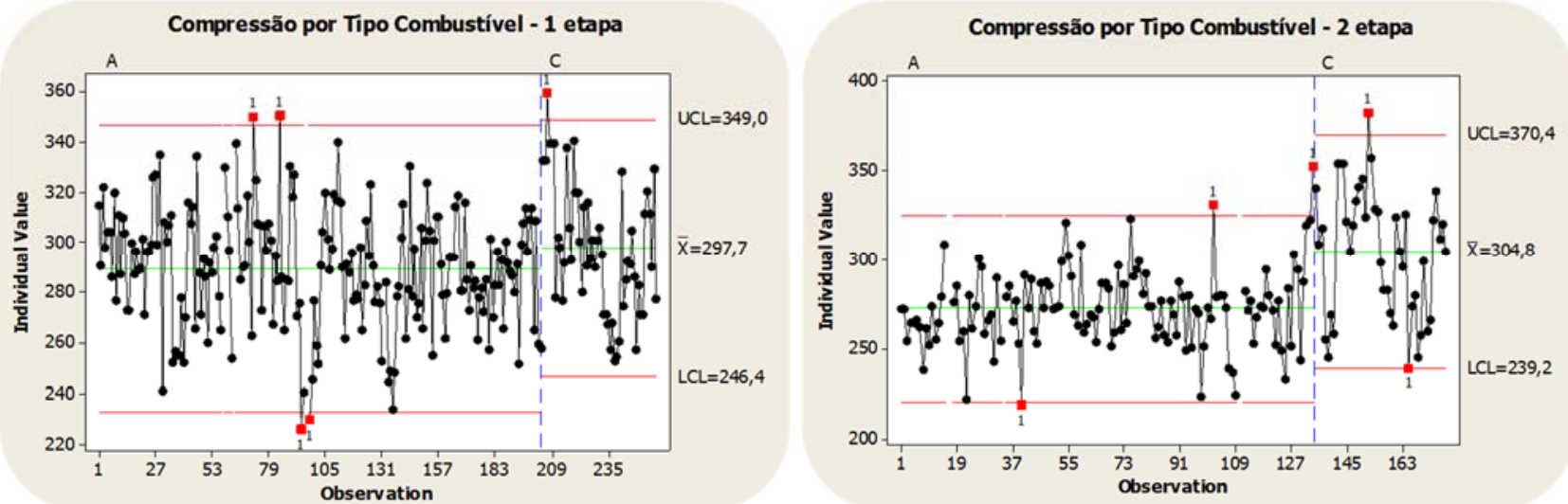

Figura 7. Gráfico de tendência por tipo de combustível sendo antracito (A) e coque breeze (C).

Com os resultados elevados de compressão obtidos foi possível elevar a dosagem de coque breeze para buscar economia no consumo de gás natural no forno.
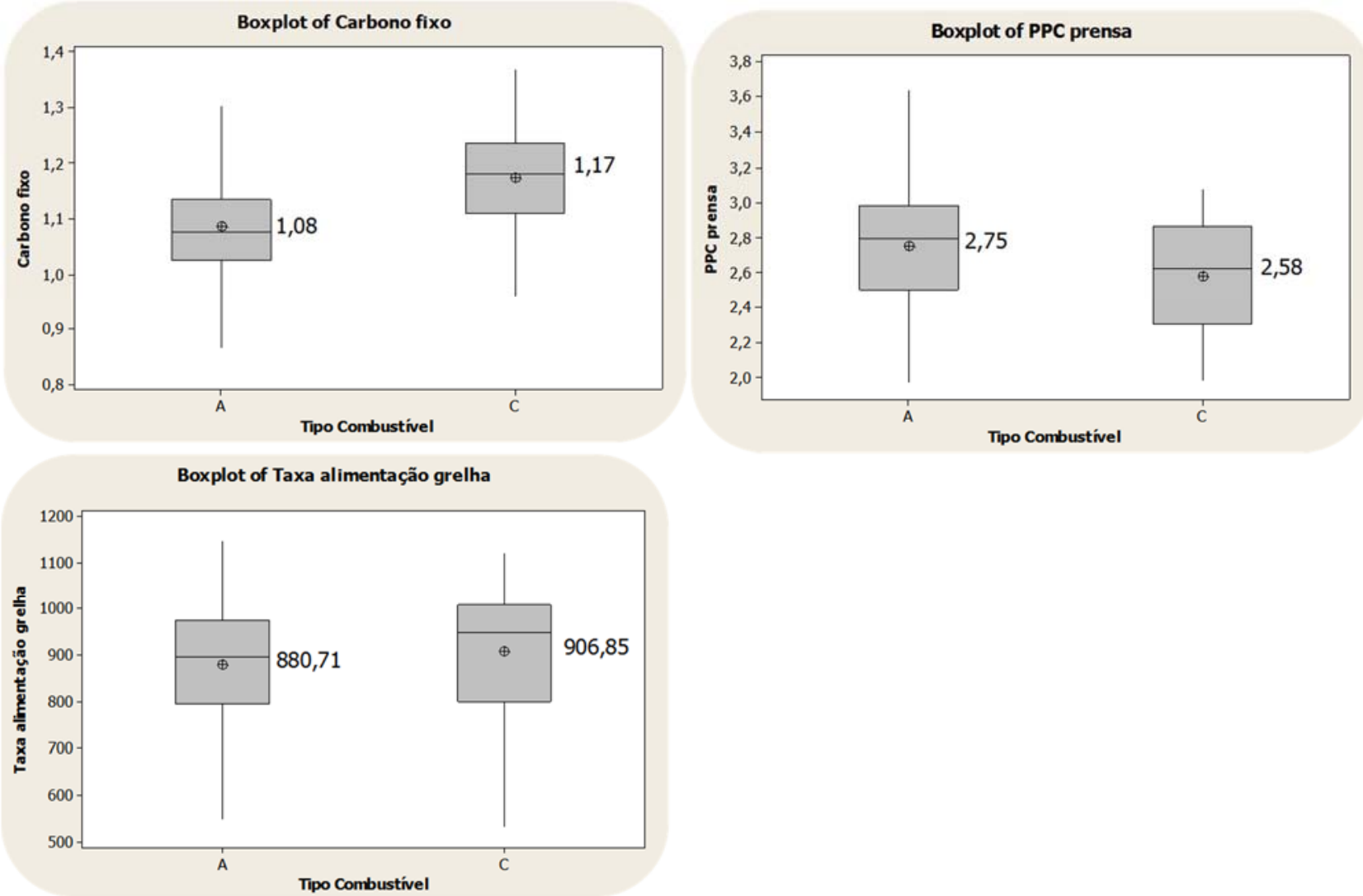

Figura 8. Boxplot para resultados operacionais do uso de antracito $(A)$ e coque breeze (C). Período compreendido entre 01/10/2014 e 01/02/2015

Com a utilização do coque breeze foi alcançada uma dosagem média de $1,17 \%$ de carbono fixo. Quando utilizado o antracito, a dosagem de carbono fixo é menor pelo impacto na compressão sendo que a dosagem utilizada depende do teor de PPC encontrado no minério prensado.

No período analisado, a taxa de alimentação da grelha média com coque foi ligeiramente maior que antracito.

A perda por calcinação do pellet feed prensado foi considerado similar para efeito de comparação entre coque breeze e antracito uma vez que os resultados estão dentro da mesma faixa de atuação para o controle de processo. 
O aumento da perda por calcinação no pellet feed causa impacto negativos em operações unitárias no processo de Pelotização o que resulta na queda da resistência a compressão da pelota.
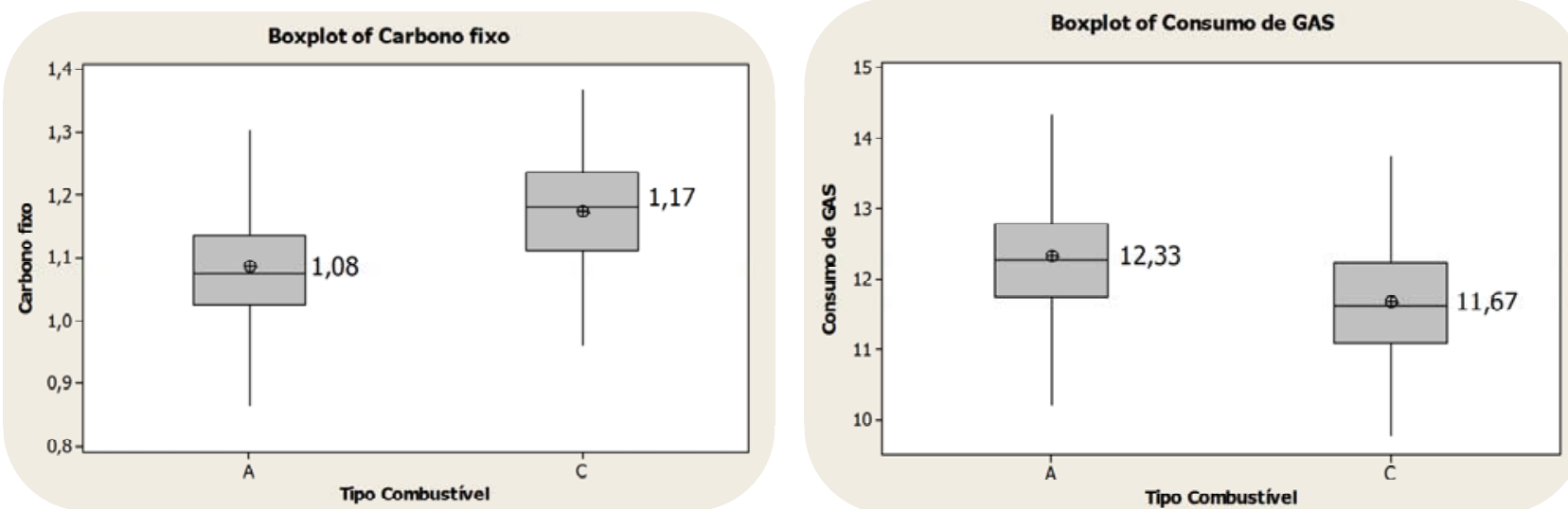

Figura 9. Boxplot para resultados de operacionais do uso de antracito (A) e coque breeze (C). Período compreendido entre 01/10/2014 e 01/02/2015

Redução do consumo de gás foi observada com uso do coque como resultado da elevação da dosagem de combustível sólido e conversão de parte da sobra de qualidade em economia de gás natural.

Nas amostras de coque analisadas no laboratório de VGR o poder calorífico médio foi similar ao do antracito e com grande variação entre as amostras enviadas.

A dosagem de teores de carbono fixo acima de 1,2\% foi limitada pela interrupção do teste e pela variabilidade da qualidade do coque breeze.

Foram obtidos períodos com valores de consumo de gás abaixo de 10,8 Nm3/t ao dosar carbono fixo acima de $1,25 \%$.
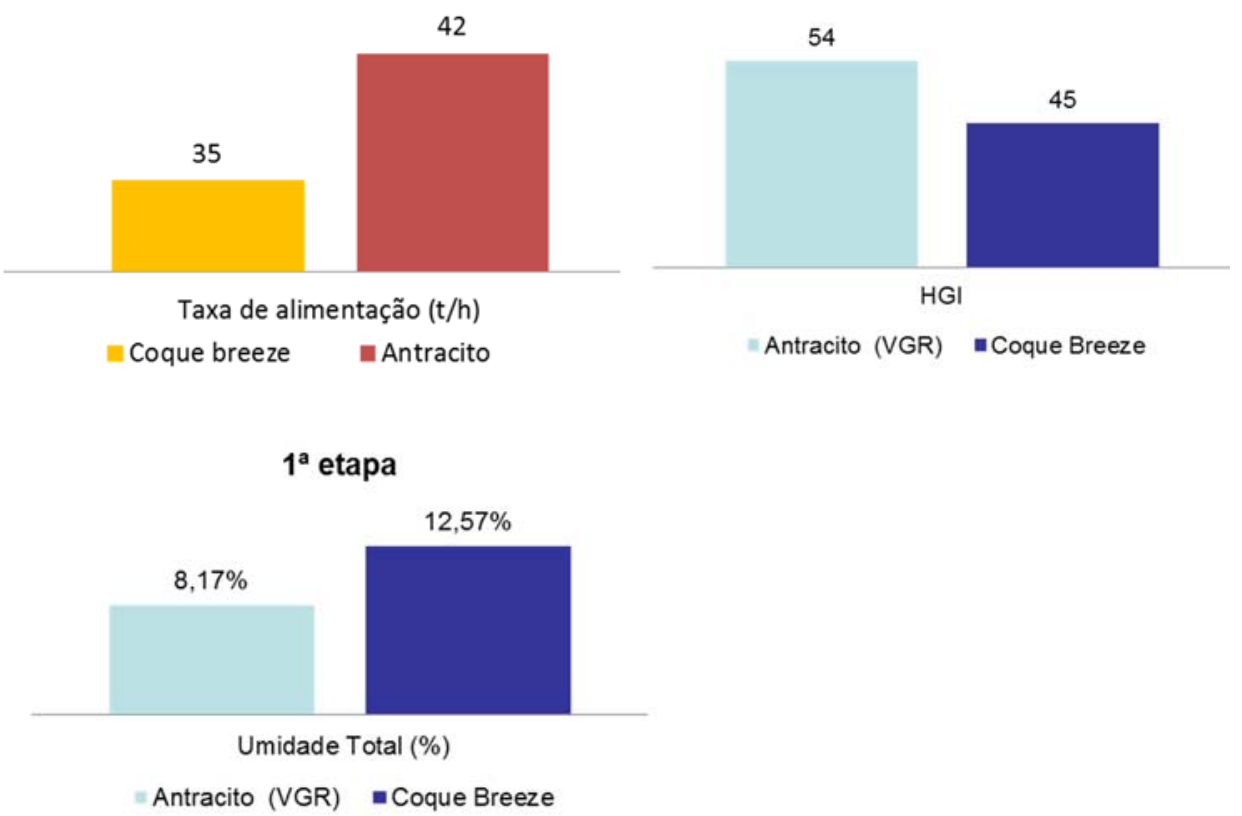

Figura 10. Taxa de alimentação, resultado de análise HGI e umidade de antracito (A) e coque breeze (C).

A taxa de alimentação do moinho 03 foi reduzida de 42t/h para aprox.35t/h. 
Ao alimentar com coque breeze ocorreu elevação da corrente do elevador de canecas e da potência de trabalho do moinho forçando redução da taxa de alimentação.

Isto era esperado uma vez que coque breeze tem menor HGI que o antracito.

Na primeira etapa do teste, a elevada umidade do coque breeze causou constantes paradas dos moinho por obstrução do sistema de alimentação o que levou a queda da taxa de utilização dos moinhos.

Ao utilizar moinho 04 para moer mix com coque ocorreu a queda da taxa de alimentação de 50t/h para 33t/h em função da limitação do sistema de transporte pneumático.

Mesmo com a queda da taxa de alimentação, a moagem de insumos é capaz de atender a demanda de MIX (coque breeze/calcário) para 7Mta de produção apenas com o moinho de rolos se utilizada a proporção percentual de coque breeze/calcário em 50/50 (considerando um rendimento operacional de $85 \%$ ). Caso seja operado com a proporção coque breeze/calcário em 40/60, isto implica em complementar a quantidade necessária de MIX operando com o moinho de bolas.

\section{Análise econômica}

\begin{tabular}{|c|c|c|c|c|}
\hline Tipo & $\begin{array}{c}\text { Preço } \\
(\mathrm{R} \$ / \mathrm{t})\end{array}$ & $\begin{array}{c}\text { Dosagem } \\
\text { cfixo }(\%)\end{array}$ & $\begin{array}{c}\% \text { cfixo no } \\
\text { combustível }\end{array}$ & $\begin{array}{c}\text { Consumo } \\
\text { de gás } \\
(\mathrm{Nm3} / \mathrm{t})\end{array}$ \\
\hline coque breeze & 415,4 & 1,17 & 77,4 & 10,57 \\
\hline antracito & 292,8 & 1,08 & 72 & 12,33 \\
\hline
\end{tabular}

Tabela 8. Dados de análise econômica do teste

PMU informado pela área de gestão de insumos.

Coque breeze com base na última aquisição, antracito e gás natural com base realizado fevereiro 2015. Valores sem impostos, com frete incluso.

Dosagem de carbono fixo: coque breeze - com base na dosagem de carbono fixo média obtida no teste; antracito - média do período utilizado para comparação neste estudo.

\% carbono fixo no combustível: coque breeze - média das amostras realizadas; antracito - média do ano. 


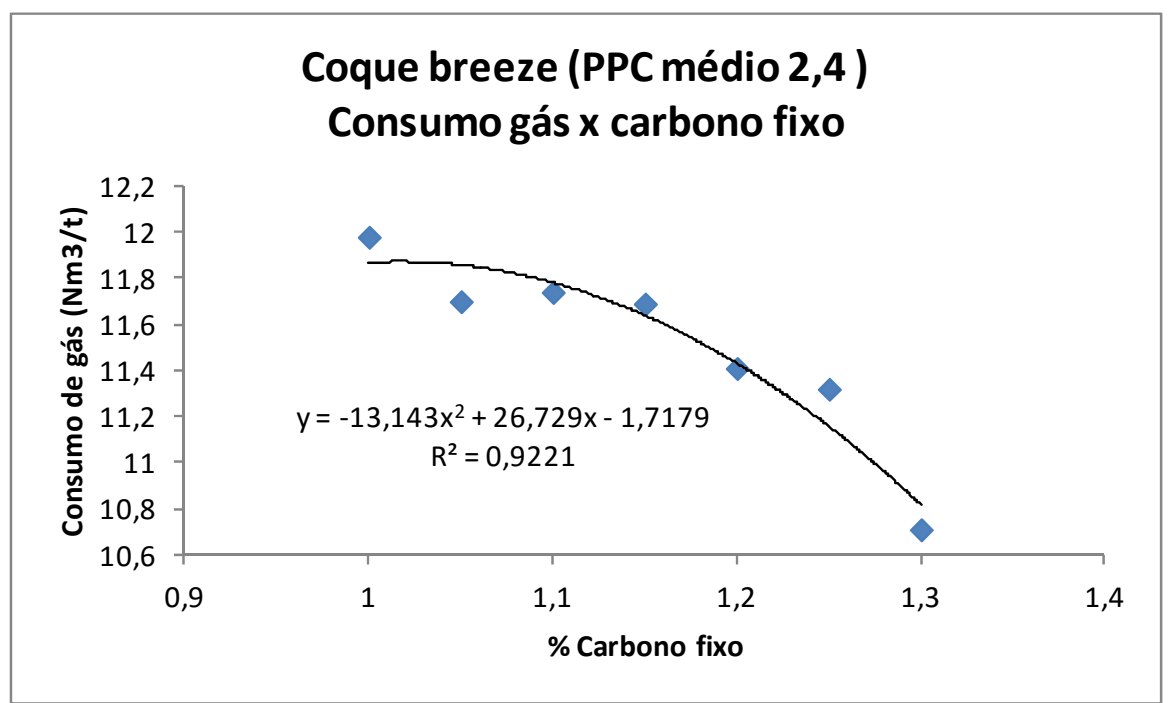

Figura 11. Gráfico de correlação entre consumo de gás natural e dosagem de carbono fixo.

Considerando dados com produção horária líquida >= $1000 \mathrm{t} / \mathrm{h}$ existe potencial para redução significativa do consumo de gás com a dosagem de teores de carbono fixo acima de $1,25 \%$ o que levaria ao equilíbrio econômico com antracito.

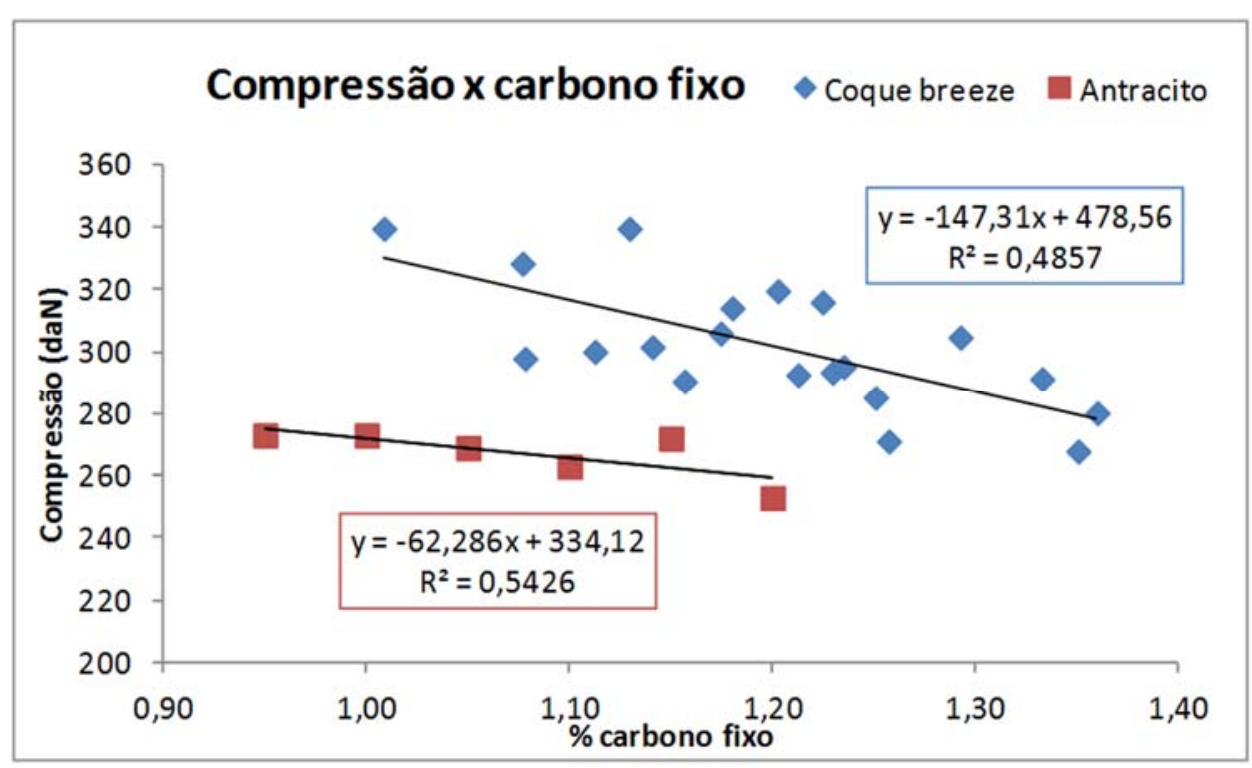

Figura 12. Gráfico de correlação entre resistência à compressão e dosagem de carbono fixo para antracito e coque breeze

Para mesma faixa de carbono fixo, os resultados de compressão com uso de coque breeze são mais altos em comparação ao uso de antracito.

Com coque breeze foi possível elevar dosagem de carbono fixo atendendo a especificação de compressão.

\section{CONCLUSÃO}

Pelas análises realizadas, o coque breeze apresenta-se contaminado com antracito e/ou carvão betuminoso o que pode ter impacto negativamente na avaliação do uso de coque breeze.

Foi observado industrialmente um ganho médio nos resultados de compressão de $20 \mathrm{daN}$. 
Foi possível elevar dosagem de carbono fixo até $1,25 \%$ sem prejuízo para qualidade com produção elevada e PPC em torno de 2,3 a $2,4 \%$.

Avaliação econômica (redução consumo de gás versus custo adicional do coque breeze) mostrou-se positiva quando se avalia resultados obtidos com a dosagem de carbono fixo acima de $1,2 \%$.

Apesar da queda de produtividade, a moagem de insumos é capaz de suprir demanda de insumos para produção da usina na capacidade nominal.

Existe viabilidade técnico-econômica positiva neste teste suficiente para a realização de um novo lote experimental com maior tempo de duração para explorar todo o potencial de ganho da utilização do coque breeze nos parâmetros de qualidade e consumo de gás natural.

Sugere-se que a especificação de compra do coque breeze seja mais rígida, principalmente em termos de teor de voláteis, carbono fixo e umidade.

\section{REFERÊNCIAS}

1 MEYER, K. - Pelletizing of Iron Ores - Springer-Verlag Berlin, 302 p,1980.

2 BALL, D.F.; DARTNELL, J.; DAVISON, J.; GRIEVE, A. e WILD, R..-Agglomeration of Iron Ores, 1973. 388 p. 